\title{
Editorial
}

\section{Is Kleine-Levin syndrome a variant of bipolar disorder? An hypothesis}

\section{Introduction}

Kleine-Levin syndrome (KLS) was first described in 1862 by Brierre de Boismont and gained its eponymous name from the reports of Willi Kleine (1) and Max Levin (2) in the 1920s. It is a rare disorder that occurs worldwide and is characterised by episodes of sleep, behavioural, mood and cognitive disturbance, with usually an onset in adolescence and a variable episodic course. The focus on hypersomnia has resulted in its characterisation as a sleep disorder (3), with hypersomnia and megaphagia being considered to be the core symptoms (4). The disorder has therefore remained in the domains of sleep medicine and neurology, with relatively little attention from psychiatry. A recent comprehensive review of 186 cases published between 1962 and 2004 (5) brought together the features of this syndrome that suggest its complexity and variety. Some similarities with primary mood disorder are obvious, and this paper proposes that there is heuristic value in considering KLS as a variant of bipolar disorder (BD). This paper does not present new data to support this conceptualisation but examines the existing information from a new perspective in order to inform future research.

\section{The clinical features of KLS and its comparison with BD}

Age of onset

The onset of KLS is usually in childhood or adolescence, with $81 \%$ of the cases beginning in the second decade of life, and the median age of onset being 15 years (5). The range of the age of onset is wide, however, ranging from 4 to 82 years. BD also often starts in childhood. While the initial diagnosis of BD is often made in the third decade, the onset is typically 10 years before the formal diagnosis (6). It has been estimated that about $60 \%$ of BD has its onset before age 20 years and $10-20 \%$ prior to 10 years $(6,7)$.
Type of onset

The onset of KLS may be insidious or acute; for the latter, a precipitating event is often reported. In the cases reviewed (5), nearly $60 \%$ reported a precipitant, with a flu-like illness or a non-specific fever being the most common. There was no evidence for a meningoencephalitis, however, and the examination of the cerebrospinal fluid was normal in all cases. The precipitant could be head injury, drug or alcohol use, sleep deprivation or psychological stress. Precipitating factors are reported less commonly in BD, but stressful events in the preceding 6 months are known to increase the risk of an episode (8), and physical disorders such as head injury, epilepsy, stroke and multiple sclerosis are know to be occasional precipitants (9). One must, of course, consider the possibility of recall bias in anecdotal case reports, which is likely to exaggerate the frequency of precipitating events, especially when a disorder begins acutely. It is interesting to note that the frequency of such precipitants diminished with subsequent episodes of KLS, being reported in only $14.5 \%$ cases (5). This is consistent with the hypothesis that recurrent episodes tend to become more autonomous and is not dissimilar to the observation in recurrent mood disorders (9). The frequency of non-specific infections prior to the onset of KLS is nevertheless noteworthy, and their significance will be discussed later in pathophysiology.

Signs and symptoms

The hallmark of an episode of KLS is hypersomnia, which is considered to be mandatory for the diagnosis. Patients report tiredness and spend a lot of time in bed, with an overwhelming desire to sleep from which they wake up spontaneously to eat or void and can be aroused but tend to be irritable or aggressive when this is done (10). Polysomnographic studies have suggested mean 


\section{Editorial}

sleep duration of about $14 \mathrm{~h}$ per day, with an excess of stage 1 and 2 sleep during the day (5). Multiple sleep latency tests have suggested a pattern similar to that of depression (10); narcolepsy-like patterns have not been reported. Hypersomnia is not an uncommon symptom of depression (11). It can be argued, therefore, that this core feature of KLS is not inconsistent with a mood disorder. Moreover, repeat episodes of KLS are characterised by fatigue rather than hypersomnia (5).

The mood disturbance of KLS is primarily irritability, which is especially manifest when the patient is denied sleep or food. Depression is present in about one half of subjects, with some patients reporting suicidal ideation. The mood disturbance generally improves with the remission of the episode. About 1 in 10 patients go on to have hypomanic symptoms, usually at the time of recovery (10), and there have been some reports of a misdiagnosis of KLS as mania (12). Irritability is a common feature of BD in children, both during the depressive and manic episodes (13), thereby suggesting a similarity with KLS. The question that arises is whether mood disturbance must be considered to be the core feature of KLS, which would reclassify it as a mood disorder rather than as a sleep disorder.

A number of behavioural disturbances are present in KLS, some of which are shared with mood disorders. The majority of patients have a craving for food and eat excessively, some to such a large extent they can be categorised as having episodes of 'megaphagia' or 'binge eating'. A minority eat less in some of the episodes. Dietary habits change with 'pica' or craving for sweets commonly described, but the behaviour is distinct from bulimia. While it is not unusual for hyperphagia or 'carbohydrate craving' to occur in conjunction with hypersomnolence in atypical depression, the eating behaviours described in KLS are unusual in primary mood disorders. Whether this is a quantitative or a qualitative change must await a systematic examination of eating behaviours in adolescents with mood disorders. Eating disorders are commonly associated with mood disorder, being reported in $13 \%$ of BD cases in one study (14).

Many patients report derealisation (24\%), a symptom not uncommonly associated with anxiety and depression in adolescence (15). Visual or auditory hallucinations $(14 \%)$ and persecutory or referential delusions $(16 \%)$ are present in fewer cases, but reminiscent of psychotic symptoms in bipolar depression or mania in a significant minority (13). Hypersexuality is another common feature, especially in men, reminiscent of mania or a mixed affective state. Compulsive behaviour is reported less commonly.
Patients with KLS, as those with BD, show cognitive deficits during the episode, with deficits in attention and memory. Long-lasting deficits have been reported in some cases of KLS, which persist between episodes (16). This is akin to the reports of persisting neuropsychological deficits in the interval between episodes (17).

In summary, the symptoms of KLS resemble those of a mixed affective state, with the atypical features of hypersomnia and altered eating behaviour being prominent but by no means exclusive (18).

\section{Duration of episodes and course}

The episodes of KLS typically last for 1-2 weeks (reported range 2.5-80 days), with a mean 6 months and a median 3.5 months, between episodes (5). The disorder appears to be self-limiting, with the median duration being 8 years and the median number of episodes 7. Prior to its termination, the disorder typically becomes milder, with reduced frequency and intensity of symptoms. BD is, on the other hand, generally regarded as a lifelong disorder, with its frequency and severity increasing with age (19). The long-term course of adolescenceonset BD is unclear, although the preliminary evidence is that it differentiates into adult $\mathrm{BD}$ $(20,21)$. The episodic and recurrent nature is shared by the two disorders.

\section{Treatment}

During the episodes, stimulants have been noted to have some benefit, particularly in reducing hypersomnia. Because the episodes of KLS are brief and self-limiting, effective treatment should prevent relapses. In this regard, only lithium has been reported to have a significantly better result than no intervention $(22,23)$. However, a few reports also suggest benefit from carbamazepine $(5,24)$. The response to lithium in a few cases is interesting, as this drug remains the most effective mood stabiliser $(25,26)$. While the evidence from studies of paediatric BD is suboptimal, lithium appears to be an important mood stabilising agent in this age group as well (13).

In the review by Arnulf et al. (5), the response rate to lithium in KLS was $41 \%$, which is impressive indeed, and presents a strong argument for considering KLS as a bipolar spectrum disorder.

\section{Pathophysiology}

Both KLS and BD are idiopathic syndromes with their pathophysiology poorly understood. In both 
disorders, the physical examination is unremarkable. KLS patients have been examined for signs of meningitis and autonomic dysfunction during the episodes, and these have been absent in most cases. While infection has been considered to be aetiological in many cases of KLS, evidence of infectious encephalitis has been lacking from cerebrospinal fluid examination. Electroencephalographic (EEG) examination has ruled out epilepsy as being the possible cause, even though nonspecific EEG abnormalities have been reported. Structural brain imaging has not shown any abnormality, but functional imaging single photon emission tomography has shown hypometabolism in the temporal and frontotemporal regions or basal ganglia in some patients $(27,28)$. Both structural and functional brain abnormalities have been noted in BD, although they are not specific to the disorder (29). Because of the prominence of sleep and eating disturbance in KLS, considerable attention has gone to pituitary and hypothalamic function. The results have been inconsistent, with no definite abnormality being established (5). Hormonal studies in BD have similarly yielded inconsistent findings. Of the two cases with primary KLS who have had a neuropathological examination reported (30), one was abnormal with multiple thalamic lesions with inflammatory cells and mild proliferation of astrocytes in the hypothalamus. While inflammatory deposits have not been reported in $\mathrm{BD}$, significant reductions in the volumes of many brain regions as well as in the number, density and/or size of neurons and glial cells have been shown, with findings in glial cells being quite consistent (31).

In summary, while there have been some leads in understanding the aetiopathogenesis of both disorders, the findings have been inconclusive, and both disorders remain poorly understood. Another approach to their understanding has been the examination of secondary cases, i.e. KLS and BD, caused by known physical disorders. KLS has been reported following brain trauma, multiple sclerosis, neoplasm as a paraneoplastic syndrome, stroke and encephalitis and in the setting of neurodevelopmental disorders (5). BD has similarly been described after all the above causes (8). The pattern of secondary KLS is very similar to that of the primary syndrome, suggesting that the 'cause' is likely a precipitant or a facilitator in vulnerable individuals.

A caveat on the similarities between the two disorders relates to genetics. It is well recognised that genetic factors are very important in the aetiology of $\mathrm{BD}$, accounting for nearly $50 \%$ of the risk for the disorder by some estimates (32). There are some families in which a single gene may have a large effect, but the majority of cases of BD are probably determined by multiple genes with small individual effects. No genes have been reproducibly identified, but there are some promising leads (33). Genetic factors appear to be important in KLS as well, with occasional familial clustering, a possible Jewish predisposition (34), and the report of an association with human leucocyte antigen (HLA) subtype DQB1*02 (35). However, one test of the hypothesis that KLS is a variant of BD would be the examination of families of KLS patients for an over-representation of mood disorders, and vice versa; although considering the rarity of the disorder, the former may be the more appropriate strategy. The cases published in the literature do not report an excess of mood disorders in KLS (5); however, systematic study of this has been lacking, partly because it has thus far not been conceptualised as a mood disorder variant.

\section{Conclusion}

This brief comparative review highlights the many similarities between KLS and adolescence-onset BD. The episodic nature and chronic recurrent course, shared symptomatology, the idiopathic nature and treatment response of the two disorders overlap. While irritability has been the dominant emotion described in KLS, depression and occasional hypomania, eating disturbance and hypersexuality suggest that it is a mixed affective state, for which reason it has been related to BD and not recurrent major depression. Until the pathophysiology of $\mathrm{BD}$ is fully understood, this proposed association will remain tentative. However, this conceptualisation has the following meaningful consequences that can guide future research:

1. Further genetic studies of KLS are warranted, with an examination of families for an excess of mood disorders and for loci reported to be abnormal in BD. On the other hand, the genetics of KLS may also inform BD investigations, e.g. the report of HLA abnormality in some patients of KLS. Even if it is eventually shown that KLS is genetically distinct from $\mathrm{BD}$, it would serve as a phenocopy and inform us about possibly shared environmental factors.

2. The role of infectious diseases as triggers for episodes of KLS is a finding that should lead to the investigation of similar triggers for mood disorders, especially in young people.

3. The importance of sleep and appetitive disturbances in KLS suggests a possible role of hypothalamic disturbance, which should receive 


\section{Editorial}

greater attention in the neuroimaging and neuropathological investigations of $\mathrm{BD}$ as well. The inflammatory response seen in the hypothalamus of one patient cannot, of course, be generalised but points to a possible postinfectious autoimmune pathogenesis, suggesting a possible line of investigation in a subset of $\mathrm{BD}$, especially with an early and atypical onset.

4. A number of developments have occurred in the pharmacotherapy of BD, which could be applied to KLS because the rarity of the disorder would not permit the investigation of novel treatments in any systematic fashion in KLS

5. The literature suggests that KLS is self-limiting, even though the episodes may recur over many years. Longitudinal examination of the episodes, using neuroimaging and polysomnographic strategies, should chart the course of the disorder, as this may provide insights into mechanisms of amelioration that may be applicable to BD.

The conceptualisation of KLS as a subtype or a variant of $\mathrm{BD}$ therefore has the ability to inform future research in understanding the pathophysiology and in identifying new treatment strategies for both disorders.

Perminder S. Sachdev

School of Psychiatry, University of New South Wales, Neuropsychiatric Institute, Prince of Wales Hospital, Sydney, Australia.

Tel: +612 3823763;

Fax: +612 93823774;

E-mail:p.sachdev@unsw.edu.au

\section{Acknowledgements}

I am grateful to Professor Gin Malhi for his comments and to Angie Russell and Lauren Norton for preparing the manuscript. There are no conflicts of interests on the part of the author.

\section{References}

1. Kleine W. Periodische schlafsucht. Monatsschr Psychiatr Neurol 1925;57:285-320.

2. Levin M. Periodic somnolence and morbid hunger: a new syndrome. Brain 1936;59:494-504.

3. Critchley M, Hoffman H. The syndrome of periodic somnolence and morbid hunger (Kleine-Levin syndrome). Br Med J 1942;1:137-139.

4. Critchley M. Periodic hypersomnia and megaphagia in adolescent males. Brain 1962;85:627-656.
5. Arnulf i, Zeitzer JM, File J, Farber N, Mignot E. Kleine-Levin syndrome: a systematic review of 186 cases in the literature. Brain 2005;128:2763-2776.

6. Loranger AW, Levine PW. Age at onset of bipolar affective illness. Arch Gen Psychiatry 1978;35:1345-1348.

7. JoYCE PR. Age of onset in bipolar affective disorder and misdiagnosis as schizophrenia. Psychol Med 1984;14:145-149.

8. Bebbington PE, Wilkins S, Jones P et al. Life events and psychosis: initial results from the Camberwell collaborative psychosis study. Br J Psychiatry 1993;162:72-79.

9. Tsuchiya KJ, Byrne M, Mortensen PB. Risk factors in relation to an emergence of bipolar disorder: a systematic review. Bipolar Disord 2003;5:231-242.

10. Reynolds CF III, Black RS, Coble P, Holzer B, Kupfer DJ. Similarities in EEG sleep findings for Kleine-Levin syndrome and unipolar depression. Am J Psychiatry 1980; 137:116-118.

11. WANG PW. Bipolar disorders: mixed states, rapid cycling, and atypical forms. J Nerv Ment Dis 2007;195:360-361.

12. Masi G, Mucci M, D’Acunto G. Kleine-Levin syndrome mimicking as mania. J Am Acad Child Adolesc Psychiatry 2007; 46:551-552.

13. Pavuluri MN, Birmaher B, Naylor MW. Paediatric bipolar disorder: a review of the past 10 years. J Am Acad Child Adolesc Psychiatry 2005;44:846-871.

14. US Department of Health and Human Services, National Institute of Mental Health. Epidemiologic catchment area (ECA) survey of mental disorders, wave I (household), 1980-1985: [United States]. ICPSR 2002;8993:1-911.

15. KRISHNAN KRR. Psychiatric and medical comorbidities of bipolar disorder. Psychosom Med 2005;67:1-8.

16. Fresco R, Giudicelli S, Poinso Y, Tatossian A, Mouren P. Kleine-Levin syndrome (recurrent hypersomnia of male adolescents). Ann Méd Psychol (Paris) 1971;1:625-668.

17. Olley A, Malhi GS, Mitchell P, Batchelor J, Lagopoulos J, Austin MP. When euthymia is just not good enough: the neuropsychology of bipolar disorder. J Nerv Ment Dis 2005; 193:323-330.

18. Berk M, Dodd S, Malhi G. Bipolar missed states: the diagnosis and clinical salience of bipolar mixed states. Aust N Z J Psychiatry 2005;39:215-221.

19. Birmaher B. Longitudinal course of paediatric bipolar disorder. Am J Psychiatry 2007;164:537-539.

20. Craney JL, Geller B. A prepubertal and early adolescent bipolar disorder-I phenotype: review of phenomenology and longitudinal course. Bipolar Disord 2003;5:243-256.

21. Cahill C, Hanstock T, Jairam R, Hazell P, Walter G, Malhi GS. Comparison of diagnostic guidelines for juvenile bipolar disorder. Aust N Z J Psychiatry 2007;41: 479-484.

22. Poppe M, Friebel D, Reuner U, Todt H, Koch R, Heubner G. The Kleine-Levin syndrome - effects of treatment with lithium. Neuropediatrics 2003;34:113-119.

23. SMolik P, Roth B. Kleine-Levin syndrome etiopathogenesis and treatment. Acta Univ Carol Med Monogr 1988;128:5-94.

24. Mukaddes NM, Kora ME, Bilge S. Carbamazepine for Kleine-Levin syndrome. J Am Acad Child Adolesc Psychiatry 1999;38:791-792.

25. Baldessarini RJ, Tondo L, Hennen J, Viguera AC. Is lithium still worth using? An update of selected recent research. Harv Rev Psychiatry 2002;10:59-75.

26. Goodwin GM, Malhi GS. What is a mood stabilizer? Psychol Med 2007;37:609-614.

27. Lu ML, Liu H, Chen CH, Sung SM. Kleine-Levin syndrome and psychosis: observation from an unusual 
Editorial

case. Neuropsychiatry Neuropsychol Behav Neurol 2000; 13:140-142.

28. Landtblom AM, Dige N, Schwerdt K, Safstrom P, Granerus G. Short-term memory dysfunction in KleineLevin syndrome. Acta Neurol Scand 2003;108:363-367.

29. Bearden CE, Hoffman KM, Cannon TD. The neuropsychology and neuroanatomy of bipolar affective disorder: a critical review. Bipolar Disord 2001;3:106-150.

30. Carpenter S, Yassa R, Ochs R. A pathologic basis for Kleine-Levin syndrome. Arch Neurol 1982;39:25-28.

31. Rajkowska G. Cell pathology in bipolar disorder. Bipolar Disord 2002;4:105-116.
32. Berrettini WH. Genetics of psychiatric disease. Annu Rev Med 2000;51:465-479.

33. Craddock N, Jones I. Molecular genetics of bipolar disorder. Br J Psychiatry 2001;178(Suppl 41):S128-S133.

34. Gadoth N, Kesler A, Vainstein G, Peled R, Lavie P. Clinical and polysomnographic characteristics of 34 patients with Kleine-Levin syndrome. J Sleep Res 2001; 10:337-341.

35. Dauvilliers Y, Mayer G, Lecendreux M et al. KleineLevin syndrome: an autoimmune hypothesis based on clinical and genetic analyses. Neurology 2002;59:17391745. 